\title{
Situational Awareness and Emergent Response Systems in the Context of Stages of Clinical Deterioration in the Hospital
}

Sashikanth Kodali

Associate Medical Director of Informatics, Vidant Health, Greenville, NC, USA.

"Corresponding author: Sashikanth Kodali, Associate medical director of informatics, Vidant Health, Greenville, NC, USA, Tel: 252-975-4100; E-mail: skodali@geisinger.edu

Received date: May 06, 2014, Accepted date: June 06, 2014, Published date: June 09, 2014

Copyright: (c) 2014 Kodali S. This is an open-access article distributed under the terms of the Creative Commons Attribution License, which permits unrestricted use, distribution, and reproduction in any medium, provided the original author and source are credited.

\section{Background}

Situation Awareness (SA) refers to an individual's "perception of the elements in the environment within a volume of time and space, the comprehension of their meaning, and the projection of their status in the near future [1]". SA failures have been very well studied in other high risk industries such as aviation that require an exceptionally high performance in a dynamic environment where safety is the primary emphasis [2]. Poor SA was found to be attributable to $88 \%$ of aviation accidents involving human errors [2]. Similarly, thousands of patients die every year in the United States due to preventable safety events [3]. Lack of SA and thereby inability to respond to critical situations in a timely manner is one of the leading causes for these safety events in health care. A shared understanding within the clinical team on current status and what's going to happen next is very important for timely intervention [4]. Hospitals employ a variety of systems to enhance this understanding and trigger appropriate emergent response for a critically ill patient.

\section{Trajectory of Clinical Deterioration}

Clinical deterioration in the hospital may empirically be divided into three stages (Figure 1). The final stage, which could be called a 'catastrophic' stage, manifests with significant derangement in vital signs and eventually leads to cardiopulmonary arrest and death. Before patients reach this catastrophic stage, there is a stage of 'rapid decline' with end organ damage and physiological derangements. Prior to this a stage of 'vulnerability' exists where a combination of factors related to the patient, the condition they are suffering from and the treatments rendered may align ominously putting them at very high risk for entering the stage of rapid decline and then onto catastrophic stage. The sooner we identify a patient on the trajectory of clinical deterioration the higher the chances of rescue. Described below are the commonly used examples of solutions currently in place in the context of these stages of clinical deterioration.

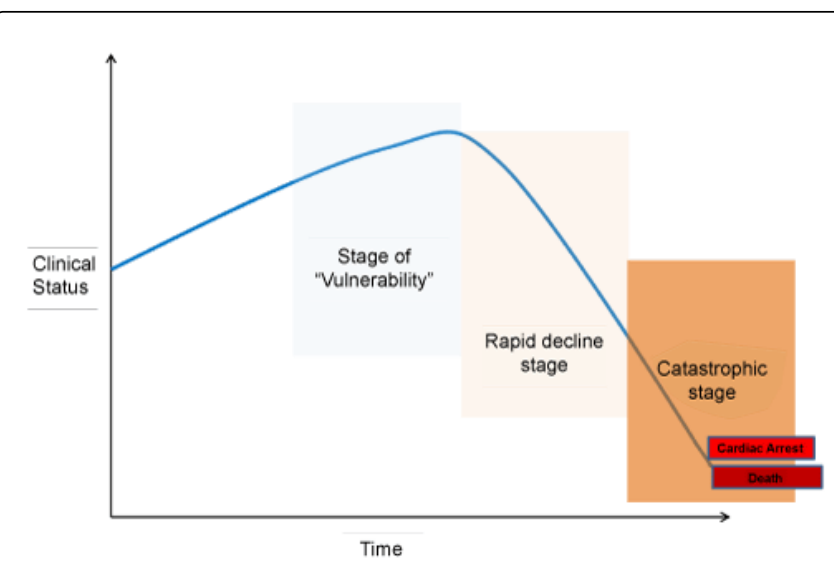

Figure 1: Three stages of clinical deterioration in a hospitalized Patient

\section{The Catastrophic Stage: Code and Rapid ResponseTeams}

The rescue outcomes of a patient in an arrest situation depend on prompt and effective chest compressions, defibrillation, assisted ventilation and appropriate medical treatment. In-hospital code teams were developed and widely adopted to respond emergently to a cardiac arrest. Although calling a code gets appropriate resuscitation resources to patient's bed side immediately, it may be too late to rescue the patients from this state [5]. This led to development of rapid response teams (RRTs) which are similar to code teams but can be summoned much earlier in the deterioration trajectory as soon as an impending cardiac arrest or other catastrophe is identified. Prior research has shown that RRTs reduce occurrence of cardiac arrests outside the ICU [6,7] and post-operative morbidity and mortality [8]. However, a recent prospective large randomized control trial involving 21 hospitals [9] and a meta-analysis of 18 studies involving 1.5 million admissions [10] did not show strong evidence for mortality reduction.

\section{The 'Rapid Decline’ Stage: Early Warning Systems}

The need for an objective triggering system which identifies patients who may be entering rapid decline stage much earlier during the hospital course led to the development of track and trigger systems such as early warning scores. Morgan et al developed an early warning score based on patient's vital signs [11]. Stenhouse et al modified the scoring system and added urinary output to develop a modified early warning score (MEWS) [12]. 
Numerous studies have validated MEWS and have shown that elevated MEWS predicts increased cardiac arrests [13], ICU admissions [14] and mortality [13,15]. These studies have led to development and implementation of treatment protocols that focus on early response for patients with high MEWS. The impact of these early response systems had been variable. In the study by Stenhouse et al, where help was sought from intensive care team when appropriate for MEWS of 4, lowered APACHE II score on admission to intensive care unit was noted suggesting early response to clinical deterioration [12]. A study by Albert et al noted 33\% reduction in cardiac arrest calls but $50 \%$ increase in rapid response team calls [16]. However, in a prospective study of 1695 acute medical admissions by Subbe et al, all patients with MEWS $>4$ were referred for critical care outreach team review, the outcomes of death, ICU admission and cardiopulmonary arrests have not changed significantly compared to an observational cohort of patients prior to intervention [17]. In another study Moon et al noted reduction of in-hospital mortality and in-hospital CPR after implementation of MEWS and critical care outreach services [18].

Early warning systems based on physiologic signs such as MEWS as described above may fail to identify many patients because of either rapid deterioration or infrequent measurement of vital signs and other physiologic parameters. A systematic review of various physiological track and trigger warning systems which looked at 36 papers that evaluated 25 systems showed their sensitivity and positive predictive value to be very low [19].

\section{The 'Vulnerability' Stage: Nursing Judgment}

Recognizing patients who are at high risk for deterioration is the most important part of SA. These patients may need escalation of care like frequent monitoring, transfer to higher level of care, consultation with specialists etc. Determining this risk requires analyzing multiple pieces of information. Although complex predictive models which are difficult to implement are being studied, the heuristics and experiential knowledge of the nurses and clinicians may play much more important role in calling attention to these patients.

The four most essential elements needed for recognition of potential for clinical deterioration are 1) Sensing- Ability to seek and gather appropriate data, 2) Perception- Collating this data into meaningful information, 3) Comprehension- Understanding the impact of the information, 4) Projection- Ability to use the information to think ahead and identify that patients are headed into catastrophic stage. Structured nurse rounding strategies like hourly rounding, intentional rounding etc are being used to improve the ability to seek and gather appropriate data. Organizing the information into carefully designed information bundles using tools like flow sheets, Kardex etc, employing questionnaires embedded in the routine assessments designed to proactively assess risk, and using structured communication strategies like Situation Background Assessment and Recommendation (SBAR) are used to improve perception and comprehension. In addition, high reliability organizations like Cincinnatti Children's hospital instituted several communication points like bedside huddles, unit based huddles, safety officer rounds etc to project harm and to systematically push and pull information through the "decision making" chain to get appropriate attention and care to the patient [20]. Further research is required in this promising area to understand how to improve and capture this 'gut feeling' of clinicians.

\section{Conclusion}

Improving situational awareness in the hospital is a complex task and requires ingenious reengineering of the care processes and communication. Although each of the solutions described in the article are useful, no single solution is effective at bringing significant reduction in morbidity and mortality outcomes. Rather, a synergistic combination of interventions that address each stage of clinical deterioration and employ both objective and subjective criteria for identification of these patients will be more effective. Understanding the distinct nature of the stages of clinical deterioration as described above will help clinical nurses and nursing leaders in designing successful solutions in this area. The readers should carefully consider the current strategies in place within their organization, evaluate any potential gaps in the culture and processes in addressing all stages of clinical deterioration and develop an appropriate action/ communication plan to bridge these gaps.

\section{References}

1. Endsley M (1995) Towards a theory of situation awareness in dynamic systems. Hum Factors 37: 32-64.

2. Singh H, Petersen LA, Thomas EJ (2006) Understanding diagnostic errors in medicine: a lesson from aviation. Qual Saf Health Care 15: 159-164.

3. Muething SE, Goudie A, Schoettker PJ, Donnelly LF, Goodfriend MA, et al. (2012) Quality improvement initiative to reduce serious safety events and improve patient safety culture. Pediatrics 130: 423-431.

4. Wright M, Endsley M (2008) Building shared situation awareness in healthcare settings. Improving healthcare team communication: Building lessons from aviation and aerospace 97-114.

5. Peberdy MA, Kaye W, Ornato JP, Larkin GL, Nadkarni V, et al. (2003) Cardiopulmonary resuscitation of adults in the hospital: a report of 14720 cardiac arrests from the National Registry of Cardiopulmonary Resuscitation. Resuscitation 58: 297-308.

6. Buist MD, Moore GE, Bernard SA, Waxman BP, Anderson JN, et al. (2002) Effects of a medical emergency team on reduction of incidence of and mortality from unexpected cardiac arrests in hospital: preliminary study. BMJ 324: 387-390.

7. DeVita MA, Braithwaite RS, Mahidhara R, Stuart S, Foraida M, et al. (2004) Use of medical emergency team responses to reduce hospital cardiopulmonary arrests. Qual Saf Health Care 13: 251-254.

8. Bellomo R, Goldsmith D, Uchino S, Buckmaster J, Hart G, et al. (2004) Prospective controlled trial of effect of medical emergency team on postoperative morbidity and mortality rates. Crit Care Med 32: 916-921.

9. Hillman K, Chen J, Cretikos M, Bellomo R, Brown D, et al. (2005) Introduction of the medical emergency team (MET) system: a clusterrandomised controlled trial. Lancet 365: 2091-2097.

10. Chan PS, Jain R, Nallmothu BK, Berg RA, Sasson C (2010) Rapid Response Teams: A Systematic Review and Meta-analysis. Arch Intern Med 170: 18-26.

11. Morgan R JM, F Williams, M M Wright (1997) An early warning scoring system for detecting developing critical illness. Clin Intensive Care 8: 100

12. Stenhouse C, Coates S, Tivey M, Allsop P, Parker T (2000) British Journal of Anaesthesia 84: 663-663.

13. Subbe CP, Kruger M, Rutherford P, Gemmel L (2001) Validation of a modified Early Warning Score in medical admissions. QJM 94: 521-526.

14. Gardner-Thorpe J, Love N, Wrightson J, Walsh S, Keeling N (2006) The value of Modified Early Warning Score (MEWS) in surgical in-patients: a prospective observational study. Ann R Coll Surg Engl 88: 571-575.

15. Quarterman CP, Thomas AN, McKenna M, McNamee R (2005) Use of a patient information system to audit the introduction of modified early warning scoring. J Eval Clin Pract 11: 133-138. 
Citation: Kodali S (2014) Situational Awareness and Emergent Response Systems in the Context of Stages of Clinical Deterioration in the Hospital . J Nurs Care 3: 171. doi:10.4172/2167-1168.1000171

Page 3 of 3

16. Albert BL, Huesman L (2011) Development of a modified early warning score using the electronic medical record. Dimens Crit Care Nurs 30: 283-292.

17. Subbe C P, Davies R G, Williams E, Rutherford P, Gemmell L (2003) Effect of introducing the Modified Early Warning score on clinical outcomes, cardio-pulmonary arrests and intensive care utilisation in acute medical admissions. Anaesthesia 58: 797-802.

18. Moon A, Cosgrove JF, Lea D, Fairs A, Cressey DM (2011) An eight year audit before and after the introduction of modified early warning score
(MEWS) charts, of patients admitted to a tertiary referral intensive care unit after CPR. Resuscitation 82: 150-154.

19. Gao H, McDonnell A, Harrison DA, Moore T, Adam S, et al. (2007) Systematic review and evaluation of physiological track and trigger warning systems for identifying at-risk patients on the ward. Intensive Care Med 33: 667-679.

20. Brady P W, Muething S, Kotagal U, AshbyM, Gallagher R et al. (2013) Improving situation awareness to reduce unrecognized clinical deterioration and serious safety events. Pediatrics 131: e298-e308. 